\title{
TIMPANOPLASTIA EN PERFORACIÓN TIMPÁNICA SECUNDARIA A TRAUMA POR ONDA EXPLOSIVA
}

\author{
Leonardo Elías Ordóñez-Ordóñez', Liliana Vitery Erazo², Néstor Ricardo González Marín", \\ Diana Patricia Parra Valencia ${ }^{3}$, Ricardo Silva Rueda ${ }^{4}$
}

${ }^{1}$ Otólogo-Otorrinolaringólogo. Servicio de Otorrinolaringología Hospital Militar Central. Clínica Universitaria Colombia. Profesor Universidad Militar Nueva Granda. Bogotá. Colombia.

${ }^{2}$ Residente Otorrinolaringología Universidad Militar Nueva Granada. Hospital Militar Central. Bogotá. Colombia. ${ }^{3}$ Otorrinolaringóloga. Ejército Nacional de Colombia. Bogotá. Colombia.

${ }^{4}$ Otorrinolaringólogo. Servicio de Otorrinolaringología Hospital Militar Central. Profesor Universidad Militar Nueva Granda. Bogotá. Colombia

Correspondencia: kokhlias@gmail.com, otoleor@gmail.com

Recibido: Enero 17 de 2014 Aceptado: Mayo 22 de 2014

\begin{abstract}
Resumen
Objetivo: Determinar si pacientes con perforación timpánica causada por trauma por onda explosiva tienen un resultado quirúrgico peor que el encontrado en pacientes con perforación timpánica causada por otitis media crónica.

Metodología: Estudio observacional analítico tipo cohorte prospectiva. Se comparó los resultados en timpanoplastia tipo I entre pacientes expuestos a trauma por onda explosiva (cohorte expuesta) y pacientes con perforación causada por otitis media crónica (cohorte no expuesta). Se incluyeron pacientes mayores de 18 años en quienes se utilizó técnica "over-under" de colocación de injerto de cartílago. Se excluyeron pacientes en quienes se utilizó otras técnicas quirúrgicas, a quienes se les practicó mastoidectomia u osciculoplastia, aquellos con perforaciones secundarias a otra etiología y quienes tenían colesteatoma. El tamaño de la muestra se calculó para la variable resultado anatómico y fue de 43 pacientes por cohorte. El estudio se terminó una vez se consiguió el tamaño de muestra propuesto. El análisis principal fue la comparación del resultado anatómico (estado del neo tímpano) y funcional (brecha aíre-hueso $<10 \mathrm{~dB}$ ) entre las cohortes expuesta y no expuesta.

Resultados: El estudio inició en agosto1 de 2011 y finalizó en julio 25 de 2013. No se encontró asociación entre la exposición bajo estudio y el fracaso anatómico, $\mathrm{RR}=4.0$ [IC 95\% RR $=0.47$ 34.35]. Tampoco hubo asociación entre el fracaso anatómico y perforación timpánica 50\%, localización anterior de la perforación timpánica e inflamación /infección al momento de la cirugía [IC 95\% RR incluyó el valor 1]. Los pacientes de la cohorte expuesta tuvieron un $\mathrm{RR}=1.76$ de fracaso funcional [IC 95\% RR=1.19-2.59].

Conclusiones: El resultado anatómico de la timpanoplastia tipo I es equiparable entre los pacientes con perforación timpánica secundaria a trauma por onda explosiva respecto a aquellos con perforaciones timpánicas por otitis crónica. Sin embargo se observó un peor resultado funcional entre los pacientes víctimas de trauma por onda explosiva.

Palabras clave: Timpanoplastia, miringoplastia, perforación de la membrana timpánica, trauma por onda explosiva, otitis media.
\end{abstract}




\title{
TYMPANOPLASTY IN TYMPANIC MEMBRANE PERFORATION CAUSED BY BLAST INJURY
}

\begin{abstract}
Objective: To determine whether patients with perforated eardrum caused by blast injury have a worse surgical outcome than that found in patients with perforated eardrum caused by chronic otitis media Methods: This is a prospective cohort study. We compared the results in type I tympanoplasty in patients exposed to blast injury (exposed cohort), and patients with perforation caused by chronic otitis media (unexposed cohort). We included patients older than 18 years in whom the over-under tympanoplasty technique was used, using cartilage graft. We excluded patients who underwent other surgical techniques, who underwent mastoidectomy and/or osciculoplastia, those with perforations secondary to other etiologies and those with cholesteatoma. The sample size was calculated for the variable anatomical outcome, and it was 43 patients per cohort. The study was finished once we reached the proposed sample size. The main outcome was the comparison of the anatomical outcome (status of the eardrum) and the functional outcome (air-bone gap $<10 \mathrm{~dB}$ ), between the exposed and unexposed cohorts.

Results: The study began in August 1, 2011 and ended on July 25, 2013. There was no association between the exposure under study and the anatomical failure, RR=4.0 [IC 95\% RR $=0.47-34.35]$. There was also no association between the anatomical outcome and size of the perforated eardrum 50\%, anterior tympanic membrane perforation and inflammation/infection at the time of surgery [IC 95\%RR included the value 1]. The patients of the exposed cohort had a $\mathrm{RR}=1.76$ for functional failure, [IC 95\% $\mathrm{RR}=1.19-2.59]$.

Conclusions: The anatomical outcome of type I tympanoplasty is comparable between patients with tympanic membrane perforation secondary to blast injury compared to those with chronic otitis media. However, we found a worse functional outcome among patients suffering from blast injury.
\end{abstract}

Key words: Tympanoplasty, myringoplasty, tympanic membrane perforation, blast injury, otitis media.

\section{TIMPANOPLASTIA EM PERFURAÇÃO TIMPÂNICA SECUNDARIA AO TRAUMA POR ONDA EXPLOSIVA}

\begin{abstract}
Resumo
Objetivo: Avaliar se os pacientes com perfuração timpânica causada pelo trauma por onda explosiva tem um resultado cirúrgico pior do que os pacientes com perfuração timpânica causada pela otites media crônica.

Metodologia: Estudo observacional analítico prospectivo. Foram comparados os resultados em timpanoplastia tipo I entre pacientes expostos ao trauma por onda explosiva (grupo exposto) e pacientes com perfuração por otites media crônica (grupo não exposto). Foram inclú́dos pacientes maiores de 18 anos utilizando a técnica "over-under" de colocação de implante de cartilagem. Foram
\end{abstract}


excluídos os pacientes que utilizaram outras técnicas cirúrgicas, mastoidectomia ou ossiculoplastia, aqueles com perfurações secundarias a outra etiologia e os que tinham colesteatoma. O tamanho dà amostra foi calculada para a variável resultado anatômico e foi de 43 pacientes por grupo. $\mathrm{O}$ Estudo finalizou no momento que a amostra foi atingida. $\mathrm{O}$ análise principal foi a comparação do resultado anatômico (estado do neo-timpano) e funcional (lacuna aire-osso $<10 \mathrm{~dB}$ ) entre os grupos exposto e não exposto.

Resultados: O estudo inicio o primeiro de agosto de 2011 e finalizou o 25 de junho de 2013. Não se apresentou associação entre a explosão no estudo e o fracasso anatômico, RR=4.0 [IC 95\% $\mathrm{RR}=0.47-34.35]$. Além disso, não houve associação entre o fracasso anatômico e perfuração timpânica $50 \%$, localização anterior da perfuração timpânica e inflamação/infecção ao momento do procedimento cirúrgico [IC 95\% RR incluiu o valor 1]. Os pacientes do grupo exposto tiveram um $\mathrm{RR}=1.76$ de fracasso funcional [IC 95\% $\mathrm{RR}=1.19-2.59$ ].

Conclusões: O resultado anatômico da timpanoplastia tipo I é equiparável entre os pacientes com perfuração timpânica secundaria ao trauma por onda explosiva quanto a aqueles com perfurações timpânicas por otites crônica. Embora, foi observado um pior resultado funcional entre os pacientes vítimas de trauma por onda explosiva.

Palavras-chave: Timpanoplastia, miringoplastia, perfuração da membrana timpânica, trauma por onda explosiva, otites media.

\section{Introducción}

La timpanoplastia tipo I (miringoplastia) es una cirugía que busca a restaurar la integridad de la membrana timpánica (MT), mejorar el mecanismo de transmisión del sonido y evitar la posibilidad de secuelas o complicaciones otológicas y/o intracraneanas (1). Aunque la causa más común de perforación de la MT es la otitis media crónica $(1,2)$, el trauma por onda explosiva puede ser una causa frecuente de perforación timpánica en países que tiene conflictos armados activos y/o son víctimas de ataques terroristas (3-6).

La perforación de la MT es la lesión primaria que con más frecuencia se encuentra en pacientes víctimas de trauma por onda explosiva $(7,8)$. La ruptura timpánica se presenta con cambios de presión menores que los que se necesitan para lesionar otros órganos que contienen aíre (pulmones y vísceras abdominales huecas), lo que explica la frecuencia de su presentación (8).

Una vez se diagnóstica una perforación timpánica, se da un periodo de tiempo para observar si hay cierre espontáneo de la misma (9), y cuando esto no ocurre el paciente es candidato para una timpanoplastia. El porcentaje de cierre espontáneo de una perforación timpánica traumática, sin importar el tipo de trauma, se ha descrito alrededor del 80\% (10). Sin embargo, en un estudio previo llevado a cabo en el Hospital Militar Central de Colombia se reportó un cierre espontáneo del $15.3 \%$, entre pacientes con perforación timpánica secundaria a trauma por onda explo- siva (4). Porcentajes de cierre espontáneo bajos también han sido descritos en otras latitudes para la perforación timpánica causada por onda explosiva. Entre pacientes víctimas de las guerras de Afganistán e Iraq se reporta un cierre espontáneo del $48 \%$ (11); y entre víctimas de una bomba en Irlanda del Norte del 38\% (12).

Las razones para estos bajos porcentajes de cierre espontáneo, en perforaciones de la MT causadas por trauma por onda explosiva, no se ha establecido claramente; sin embargo tres factores podrían tenerse en cuenta. 1. Lesión térmica del remanente timpánico, con disminución de su capacidad reparativa. 2. Contaminación del oído medio por restos de vegetales y/o tierra, propios del campo de batalla, y/o mal manejo inicial (lavado de oído con agua), con la consiguiente sobreinfección y perpetuación de la perforación timpánica (9). 3. Presencia de perforaciones de mayor tamaño, debido a la cantidad de energía liberada por los artefactos explosivos, que tienen menor posibilidad de cierre espontáneo (9).

Una posibilidad que puede plantearse razonablemente, al discutir los anteriores hallazgos, es si estos pacientes pueden tener también un peor resultado quirúrgico en razón de los factores que disminuyen el porcentaje de cierre espontáneo. Al revisar la literatura sobre el tema (Búsqueda en PubMed, febrero 25 de 2011, términos: "Tympanoplasty" [Mesh] AND "Tympanic Membrane Perforation" [Mesh]; sin límites), son pocos los estudios que han abordado este problema de investigación. En un estudio hecho entre víctimas de la guerra de Croacia, reportan un 
porcentaje de éxito de la timpanoplastia de alrededor del 90\% (13). Sin embargo este es un estudio descriptivo, sin grupo control y el resultado quirúrgico lo evalúan solamente a través de la integridad del injerto (sin tener en cuenta el resultado funcional). Otros estudios sobre el tema sólo mencionan el porcentaje de pacientes que requirió cirugía (sin mencionar porcentajes de éxito), o se enfocan en las características clínicas de este tipo de lesiones $(3,6,11,14)$.

Con el objetivo de determinar si pacientes con perforación timpánica causada por trauma por onda explosiva tienen un resultado quirúrgico peor que el encontrado en pacientes con perforación timpánica causada por otitis media crónica, se realizó el presente estudio.

\section{Materiales y métodos}

Se realizó un estudio observacional analítico tipo cohorte prospectiva, con el fin de comparar los resultados en timpanoplastia tipo I entre pacientes expuestos a trauma por onda explosiva, Grupo I (GI, cohorte expuesta), y pacientes no expuestos (perforación causada por otitis media crónica), Grupo II (GII). La investigación inició en agosto 1 de 2011 y se terminó cuando se consiguió el tamaño de muestra propuesto.

Adoptamos las directrices para reportar estudios observacionales, diseño de cohorte, STROBE (sigla del inglés "Strengthening the Reporting of Observational studies in Epidemiology") (15).

\section{Pacientes}

Se incluyeron pacientes mayores de 18 años que fueron operados de timpanoplastia tipo I utilizando técnica medial u "over-under" de colocación de injerto de cartílago. Las perforaciones timpánicas fueron causadas por trauma por onda explosiva (cohorte expuesta, GI) u otitis media crónica (cohorte no expuesta, GII).

Se excluyeron pacientes en quienes se utilizó técnica transperforación o lateral de colocación del injerto, utilización de injerto diferente a cartílago, a quienes se les practicó algún de tipo de mastoidectomia y/u osciculoplastia, aquellos con perforaciones secundarias a otra etiología, quienes tenían colesteatoma y aquellos que no desearon participar en el estudio.

Los pacientes elegibles fueron identificados a partir de los libros de programación de cirugía del servicio de Otorrinolaringología del Hospital Militar Central. Los pacientes que cumplieron los criterios de inclusión y exclusión fueron invitados a participar en el estudio durante la consulta prequirúrgica y se constituyó una cohorte expuesta (perforación timpánica causada por trauma por onda explosiva) y una cohorte no expuesta (perforación timpánica causada por otitis media crónica). A los pacientes se les explicó verbalmente los objetivos de la investigación y se les solicitó su participación voluntaria, quienes aceptaron firmaron un consentimiento diseñado para la investigación. A los participantes se les explicó claramente que su participación o no en el estudio no afecta el tratamiento que requieren, y que si lo desean pueden retirarse de la investigación en el momento que lo deseen.

Los pacientes ingresados al estudio fueron seguidos de forma prospectiva y la información se registró a medida que se presentaron los sucesos relevantes para la investigación. La información prequirúrgica se obtuvo durante la consulta prequirúrgica, la información quirúrgica fue obtenida el día de la cirugía, y la evaluación del resultado quirúrgico se registró en la consulta de control postoperatorio de los seis meses ( \pm 1 mes). También se registraron, cuando se presentaron, eventos relevantes como complicaciones durante todo el periodo del estudio.

La investigación fue aprobada por el comité de revisión institucional del Hospital Militar Central.

\section{Técnica quirúrgica}

En todos los casos se utilizó técnica "over-under" de timpanoplastia, con injerto de cartílago. La técnica "over-under" es una modificación de la técnica medial en la cual se libera el mango del martillo del remanente timpánico, y se utiliza ésta porción del martillo para ayudar a sostener el injerto en posición (16). Aunque esta técnica fue descrita con fascia temporal, en la institución del estudio se ha modificado para utilizar injerto de cartílago, y fue la forma en que todos los pacientes del presente estudio fueron operados, Figura 1.

\section{Evaluación de desenlace}

Para evaluar los resultados de la timpanoplastia se tuvo en cuenta el resultado anatómico (estado del neo tímpano) y el resultado funcional (pruebas audiométricas). Se definió como éxito anatómico la presencia de un neo tímpano íntegro, en posición normal y sin retracción. Se consideró fracaso anatómico en presencia de re perforación y/o retracción del neo tímpano. El éxito funcional se definió como el cierre de la brecha entre las vías aérea y ósea (BAH) a $10 \mathrm{~dB}$ en el promedio de tonos puros (PTP) de 


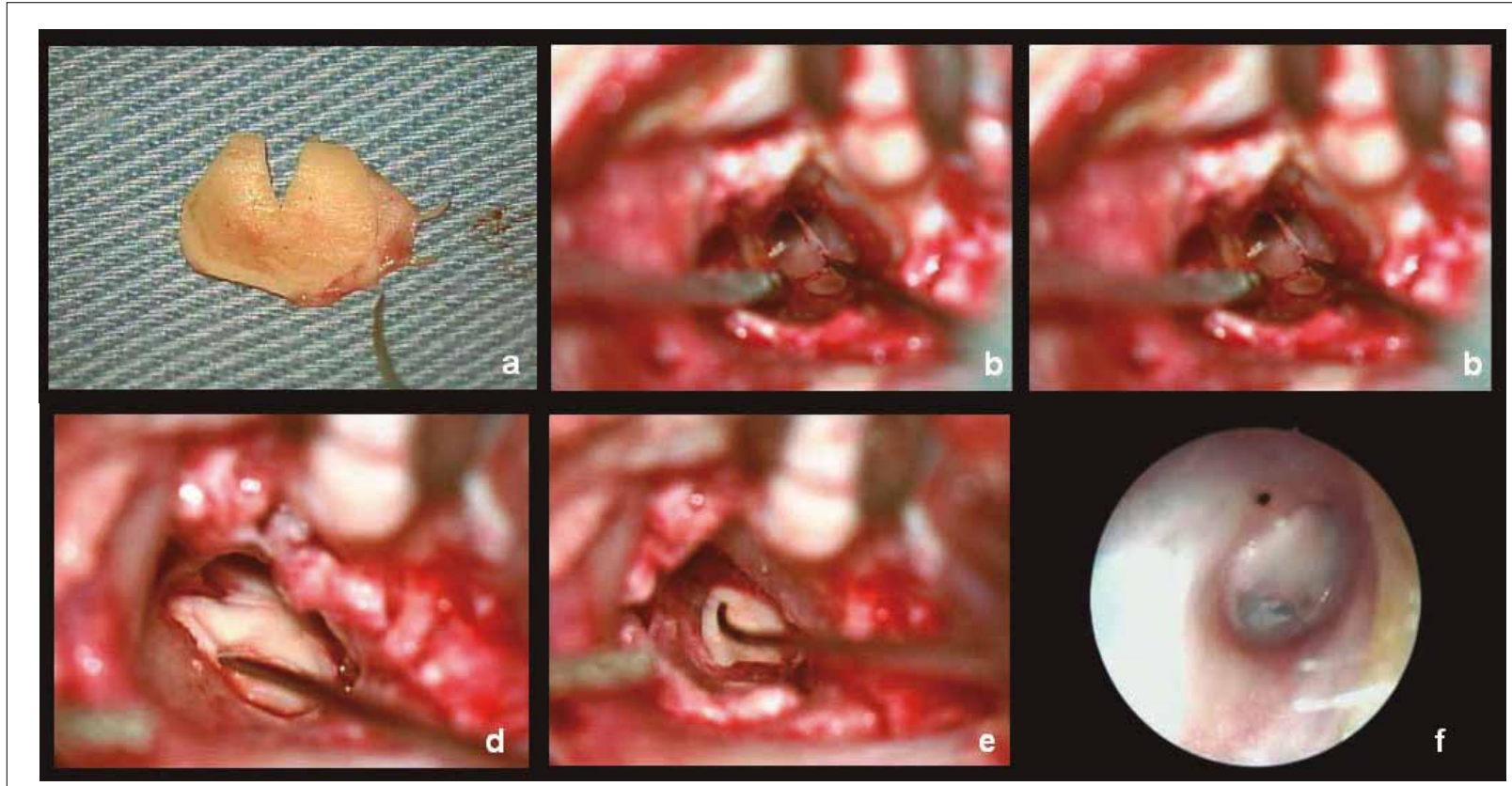

a. Una vez hecho el abordaje retroauricularse toma un injerto de cartílago de concha cimba, el cual se adelgaza y recorta en forma de un plato ovalado de unos 9 × $10 \mathrm{~mm}$ con una escotadura para alojar el mango del martillo.

b. Se hacen incisiones sobre la piel posterior del CAE, se exponen los bordes de la perforación y se reavivan sus bordes.

c. Después de elevado el colgajo timpanomeatal posterior, se ingresa al oído medio y de manera cuidadosa se libera el mango del martillo de la membrana timpánica hasta el nivel de la apófisis corta.

d. El injerto se coloca apoyándolo sobre el mango del martillo, verificando su adecuada orientación. Se coloca Gelfoam ${ }^{\circledR}$ (Upjohn Laboratories, Kalamazoo, MI) en el oído medio para sostener el injerto en contacto con el remanente timpánico.

e. Posteriormente se reposiciona el colgajo timpanomeatal y se verifica la adecuada posición del injerto en relación con los bordes de la perforación. Se coloca Gelfoam ${ }^{\circledR}$ sobre el remanente timpánico para asegurar el contacto con el injerto subyacente. Las incisiones se cierran por planos y se coloca un apósito sobre la herida. En el seguimiento postoperatorio se retira el apósito en los tres primeros días y el Gelfoam ${ }^{\circledast}$ del CAE se deja que se extruya o se retira después del primer mes.

f. Imagen postoperatoria que muestra un neo tímpano con injerto de cartílago, integro y en adecuada posición.

Figura 1. Técnica de timpanoplastia "over-under" con cartílago

cuatro frecuencias $(0.5,1,2$ y $3 \mathrm{KHz})$. En la evaluación funcional también se analizó el valor neto de la BAH y del cierre de la $\mathrm{BAH}$, según lo recomienda la Academia Americana de Otorrinolaringología y Cirugía de Cabeza y Cuello en sus guías (17). Se evaluó también el cierre de la $\mathrm{BAH}$ usando como punto de corte $20 \mathrm{~dB}$. La evaluación de los desenlaces se hizo a los seis meses ( \pm 1 mes) del seguimiento postoperatorio.

\section{Tamaño de la muestra}

El tamaño de muestra se calculó para la variable resultado anatómico (probabilidad de éxito quirúrgico). Los parámetros utilizados fueron: error tipo I ( $)=0.05$, poder $=0.8$, probabilidad de éxito quirúrgico en la cohorte de no expuestos $=0.97$ (tomado de un estudio previo realizado en la institución) (18) y probabilidad de éxito quirúrgico en la cohorte de expuestos=0.77 (utilizando una diferencia mínima clínicamente significativa del 20\%). El tamaño calculado fue de 43 pacientes para cada cohorte. El cálculo se realizó con el programa PS (v.3.0; DuPont WD, Plummer WD., Vanderbilt University, Nashville, TN, USA).

\section{Análisis estadístico}

La descripción de las variables se hizo mediante medidas de tendencia central y dispersión para las variables cuantitativas. Para las variables categóricas se hizo descripción de frecuencias a través de proporciones. 
Para las variables con distribución normal (prueba de Kolmogorov-Smirnov) se utilizó pruebas paramétricas. Para variables sin distribución normal (que se asumen como variables ordinales) y para aquellas nominales $\mathrm{u}$ ordinales se utilizaron pruebas no-paramétricas. Se buscaron diferencias entre los grupos y el nivel de significación estadística fue establecido en 0.05 , para pruebas de dos colas.

El análisis principal es la comparación del resultado anatómico y funcional de la timpanoplastia entre GI vs.GII. Además se realizará un análisis multivariado entre el resultado quirúrgico y las variables demográficas y clínicas registradas, ajustadas por las variables que presenten relación significativa en el análisis bivariado. Para el GI se determinará también la frecuencia de trauma acústico, definido como un PTP por vía ósea mayor a $15 \mathrm{~dB}$.

Como medida de asociación se calculó el riesgo relativo con su IC 95\%. Para esto se calculó la incidencia acumulada de fracaso en cada grupo y la relación entre éstas, obteniendo el riesgo de fracaso en pacientes expuestos en relación con los no expuestos.
Los análisis estadísticos se realizaron con el programa SPSS (v.11.5; SPSS, Inc., Chicago, IL, USA).

\section{Resultados}

Este estudio se realizó en el servicio de Otorrinolaringología del Hospital Militar Central, una institución de tercer nivel en Bogotá, Colombia. El estudio inició el primero de agosto de 2011 y finalizó en julio 25 de 2013, cuando se terminó el seguimiento de los pacientes.

En total se encontraron 112 pacientes elegibles, de ellos 109 se seleccionaron para el estudio y fueron asignados a la cortes expuesta $(n=56)$ y no expuesta $(n=53)$. La principal causa de exclusión fue la pérdida del seguimiento $(n=15)$, seguido por modificaciones en la técnica quirúrgica debido a hallazgos intraoperatorios $(n=8)$, Figura 2 .

Se realizó la prueba de normalidad de Kolmogorov-Smirnov para las variables proporcionales/interválicas del estudio y se encontró que no estaban normalmente distribuidas $(p<0.05)$, las variables: edad en el grupo $(p=0.039)$, distancia al punto

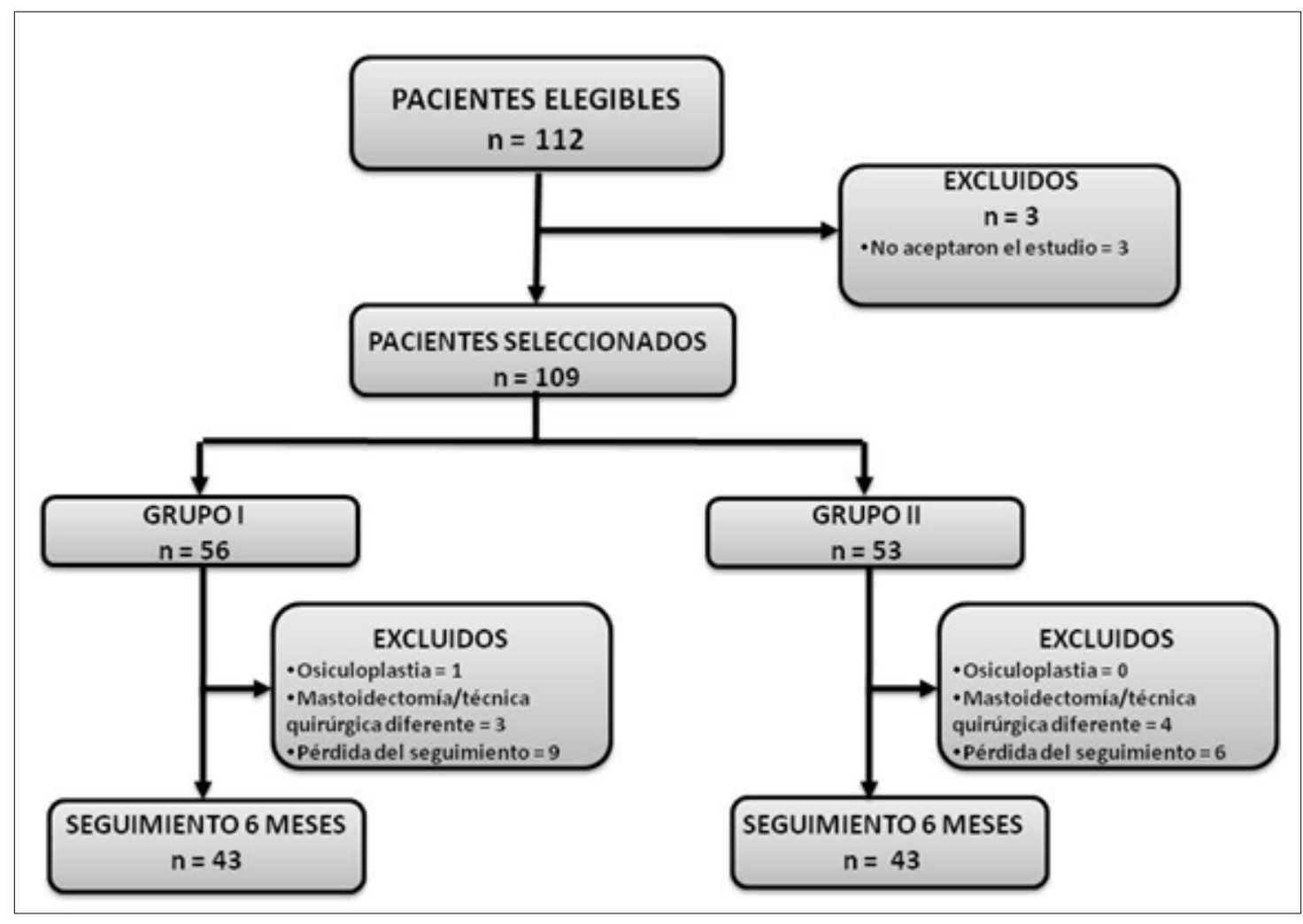

Figura 2. Flujograma de los pacientes del estudio 
de la explosión ( $p=0.034$ ), PTP vía ósea preoperatoria ( $p=$ 0.021 ), umbral de recepción del habla (URH, equivalente al SRT "speech reception threshold" de la literatura en inglés) preoperatorio $(p=0.005)$, tamaño de la perforación timpánica $(p=0.028)$, PTP vía aérea postoperatoria $(p=0.003)$, PTP vía ósea postoperatoria $(p=0.001)$, URH postoperatorio $(p<0.001)$ y porcentaje de discriminación del lenguaje (PDL, equivalente del SDS "speech discrimination score" de la literatura en inglés) postoperatorio.

En la Tabla 1 se muestran las características demográficas comparativas de los sujetos del estudio, la diferencia en el sexo entre GI vs. GII se debe a que la mayoría de los integrantes de las fuerzas armadas de Colombia son hombres.

En la Tabla 2 se presenta información relativa a los pacientes del Grupo I; la mayoría de los pacientes pertenecen al Ejército nacional (81.4\%), el tipo de artefacto que causó el trauma con más frecuencia fue la mina antipersonal (30.2\%) y los síntomas postraumáticos más frecuentes fueron hipoacusia (86\%) y tinitus (86\%).La lesión adicional más frecuente fue el trauma ocular, en ocho pacientes (18.6\%), de los cuales tres (7\%) tuvieron pérdida visual completa. 28 pacientes (65.1\%) tuvieron perforación timpánica aislada. Las fechas en que ocurrió el evento traumático para los pacientes del grupo I estuvieron comprendidas entre junio 15 de 1991 hasta septiembre 26 de 2012. La distancia promedio entre el paciente y la fuente del trauma fue 3.1 metros $(\mathrm{DE}=3.7 \mathrm{~m}$. Mínimo $=0.1 \mathrm{~m}$ y Máximo $=20 \mathrm{~m})$. En 22 pacientes (51.2\%) hubo sobreinfección bacteriana, ya sea por contaminación durante el evento traumático y/o mal manejo de la perforación (lavado de oído con agua). 24 pacientes de este grupo (55.8\%) recibieron algún tipo de tratamiento inicial (antibiótico tópico y/o sistémico), aunque solo 18 (41.8\%) refirieron haber recibido la recomendación de evitar la entrada de agua en los oídos.

Tabla 1. Línea de base de los sujetos de la cohorte de estudio.

\begin{tabular}{|l|c|c|c|c|}
\hline & $\begin{array}{c}\text { Cohorte } \\
\text { expuesta }\end{array}$ & $\begin{array}{c}\text { Cohorte no } \\
\text { expuesta }\end{array}$ & Total & p \\
\hline No. de pacientes & 43 & 43 & 86 & NA \\
\hline $\begin{array}{l}\text { Edad (años): } \\
\text { Mediana [RIC] }\end{array}$ & $26[10]$ & $30[24]$ & $27[13.3]$ & $0.193 \mathrm{a}$ \\
\hline $\begin{array}{l}\text { Sexo: } \\
\text { Hombres/Mujeres }\end{array}$ & $43 / 0$ & $23 / 20$ & $66 / 20$ & $<0.001 \mathrm{~b}$ \\
\hline $\begin{array}{l}\text { Lado de la cirugía: } \\
\text { Derecho/Izquierdo }\end{array}$ & $21 / 22$ & $22 / 21$ & $43 / 43$ & $0.83 \mathrm{c}$ \\
\hline
\end{tabular}

NA: No aplica. RIC: recorrido intercuartil.

a Prueba U de Mann-Whitney

b Prueba exacta de Fisher

c Prueba de Chi cuadrado
Tabla 2. Caracterización de los pacientes con perforación timpánica causada por trauma por onda explosiva (Cohorte expuesta)

\begin{tabular}{|c|c|}
\hline Variable & $\begin{array}{c}\text { No. de } \\
\text { pacientes } \\
(\mathrm{n}=43) \\
\mathrm{n}(\%)\end{array}$ \\
\hline \multicolumn{2}{|l|}{ Fuerza Militar } \\
\hline Ejército Nacional & $35(81.4 \%)$ \\
\hline Fuerza Aérea & $0(0 \%)$ \\
\hline Armada Nacional & $8(18.6 \%)$ \\
\hline \multicolumn{2}{|l|}{ Tipo de Trauma } \\
\hline Mina antipersonal & $14(32.6 \%)$ \\
\hline Bomba & $11(25.6 \%)$ \\
\hline Polígono & $9(20.9 \%)$ \\
\hline Granada & $5(11.6 \%)$ \\
\hline Uso de arma en combate & $3(7 \%)$ \\
\hline Trauma por rayo & $1(2.3 \%)$ \\
\hline \multicolumn{2}{|l|}{ Tinitus } \\
\hline $\mathrm{Si}$ & $37(86 \%)$ \\
\hline \multicolumn{2}{|l|}{ Hipoacusia } \\
\hline $\mathrm{Si}$ & $37(86 \%)$ \\
\hline \multicolumn{2}{|l|}{ Otorragia } \\
\hline $\mathrm{Si}$ & $18(41.9 \%)$ \\
\hline \multicolumn{2}{|l|}{ Otalgia } \\
\hline $\mathrm{Si}$ & $30(69.8 \%)$ \\
\hline \multicolumn{2}{|l|}{ Disacusia/hiperacusia } \\
\hline $\mathrm{Si}$ & $4(9.3 \%)$ \\
\hline \multicolumn{2}{|l|}{ Vértigo } \\
\hline $\mathrm{Si}$ & $19(44.2 \%)$ \\
\hline \multicolumn{2}{|l|}{ Otras lesiones corporales } \\
\hline Trauma ocular con pérdida visual & $3(7 \%)$ \\
\hline Trauma ocular sin pérdida visual & $5(11.6 \%)$ \\
\hline Lesión en piel/tejido blando llevado a cirugía & $7(16.3 \%)$ \\
\hline Fractura de extremidad (es) & $5(11.6 \%)$ \\
\hline Amputación parcial/total extremidad (es) & $1(2.3 \%)$ \\
\hline Trauma abdominal llevado a cirugía & $1(2.3 \%)$ \\
\hline Trauma de tórax llevado a cirugía & $2(4.7 \%)$ \\
\hline Trauma craneoencefálico & $1(2.3 \%)$ \\
\hline Trauma facial llevado a cirugía & $3(7.0 \%)$ \\
\hline Trauma genital & $1(2.3 \%)$ \\
\hline
\end{tabular}

El PTP postraumático por vía ósea para los pacientes de $\mathrm{Gl}$ fue de $32.5 \mathrm{~dB}$ ( $\mathrm{DE}=7.2$ ), encontrando que 19 de ellos (44.2\%) tuvieron un PTP $15 \mathrm{~dB}$, lo que se interpreta como trauma acústico en las frecuencias del habla.

En la Tabla 3 se presentan los hallazgos quirúrgicos para los dos grupos. Observamos que los pacientes de GI presentaron perforaciones de mayor tamaño y que con más frecuencia comprometieron los cuadrantes anterio- 
Tabla 3. Hallazgos quirúrgicos en los pacientes del estudio.

\begin{tabular}{|c|c|c|c|c|}
\hline $\begin{array}{l}\text { Hallazgos } \\
\text { quirúrgicos }\end{array}$ & $\begin{array}{c}\text { Cohorte } \\
\text { expuesta } \\
\mathbf{n}=43\end{array}$ & $\begin{array}{c}\begin{array}{c}\text { Cohorte } \\
\text { no }\end{array} \\
\text { expuesta } \\
n=43\end{array}$ & $\begin{array}{c}\text { Total } \\
\mathrm{n}=86\end{array}$ & $\mathbf{p}$ \\
\hline \multicolumn{5}{|c|}{ Perforación cuadrantes anteriores (n) } \\
\hline $\mathrm{Si}$ & 30 & 19 & 49 & \multirow{2}{*}{$0.017^{\mathrm{a}}$} \\
\hline No & 13 & 24 & 37 & \\
\hline \multicolumn{5}{|c|}{ Tamaño de perforación } \\
\hline Mediana [RIC] & $60[40]$ & $40[40]$ & $50[40]$ & $0.047^{\mathrm{b}}$ \\
\hline \multicolumn{5}{|c|}{ Timpanoesclerosis (n) } \\
\hline $\mathrm{Si}$ & 5 & 6 & 11 & \multirow{2}{*}{$0.75^{\mathrm{a}}$} \\
\hline No & 38 & 37 & 75 & \\
\hline \multicolumn{5}{|c|}{ Inflamación/infección en cirugía (n) } \\
\hline $\mathrm{Si}$ & 9 & 5 & 14 & \multirow{2}{*}{$0.24^{\mathrm{a}}$} \\
\hline No & 34 & 38 & 72 & \\
\hline
\end{tabular}

RIC: Recorrido intercuartil

a Prueba de chi cuadrado

b Prueba U de Mann-Whitney

res, diferencias que fueron estadísticamente significativas $(p<0.05)$.

El análisis del resultado anatómico se presenta en la Tabla 4, no se encontró asociación entre la exposición bajo estudio (perforación timpánica causada por trauma por onda explosiva) y el fracaso quirúrgico de la timpanoplastia tipo I (IC 95\% RR= 0.47-34.35). Tampoco hubo asociación entre el fracaso quirúrgico y perforación de la MT 50\%, localización anterior de la perforación de la MT e inflamación /infección al momento de la cirugía (IC 95\% RR incluyó el valor 1). El porcentaje de éxito quirúrgico para la cohorte expuesta fue de $90.1 \%$ (39/43) y en la cohorte no expuesta de $97.7 \%(42 / 43)$.

Respecto al resultado funcional, se presenta en las Tabla 5, 6 y 7. En la Tabla 5 se muestra la línea de base audiométrica (valores preoperatorios), se observa que el PTP por vía aérea y la $\mathrm{BAH}$ fue significativamente mayor en la cohorte expuesta.

Los resultados funcionales dentro de cada cohorte se presentan en la Tabla 6, se observa que para las dos cohortes hay una disminución significativa en el valor postoperatorio respecto al preoperatorio para las variables PTP por vía área, BAH y URH ( $p<0.05)$; lo que implica una mejoría significativa en la hipoacusia conductiva con la cirugía. No hubo cambio significativo para las variables PTP por vía ósea y PDL entre el postoperatorio y el preoperatorio, para las dos cohortes ( $p>0.05$ ); que se interpreta como ausencia de daño en la función del oído interno con la cirugía.

En la Tabla 7 se presentan los resultados funcionales comparativos, hubo una diferencia significativa entre la cohorte expuesta y no expuesta cuando para medir el resultado funcional se utilizó el cierre de la $\mathrm{BAH}$ con un punto de corte en $10 \mathrm{~dB}(p=0.017)$, pero no cuando se utilizó el punto de corte en $20 \mathrm{~dB}(p=0.71)$. El riesgo relativo de fracaso funcional en la cohorte expuesta fue $\mathrm{RR}=1.76$ [IC

Tabla 4. Resultado anatómico de los pacientes del estudio

\begin{tabular}{|c|c|c|c|c|c|}
\hline Variable & $\begin{array}{c}\text { Fracaso } \\
\text { (n) }\end{array}$ & $\begin{array}{l}\text { Éxito } \\
\text { (n) }\end{array}$ & $\mathbf{R R}$ & IC $95 \%$ RR & $\mathbf{p}$ \\
\hline \multicolumn{6}{|l|}{ Etiología } \\
\hline Cohorte Expuesta (Trauma por onda explosiva) & 4 & 39 & \multirow[t]{2}{*}{4.0} & \multirow{2}{*}{$0.47-34.35$} & \multirow{2}{*}{$0.36^{\mathrm{a}}$} \\
\hline Cohorte No Expuesta (Otitis media crónica) & 1 & 42 & & & \\
\hline \multicolumn{6}{|l|}{ Tamaño de la perforación timpánica 50\% } \\
\hline $\mathrm{Si}$ & 3 & 47 & 1.08 & $0.19-6.14$ & $1.0^{\mathrm{a}} \mathrm{a}$ \\
\hline No & 2 & 34 & & & \\
\hline \multicolumn{6}{|l|}{ Localización anterior de la perforación timpánica } \\
\hline $\mathrm{Si}$ & 4 & 45 & 0.33 & $0.04-2.84$ & $0.39^{\mathrm{a}}$ \\
\hline No & 1 & 36 & & & \\
\hline \multicolumn{6}{|l|}{ Inflamación/infección al momento de la cirugía } \\
\hline $\mathrm{Si}$ & 2 & 12 & 3.43 & $0.63-18.68$ & $0.19^{\mathrm{a}}$ \\
\hline No & 3 & 69 & & & \\
\hline \multicolumn{6}{|l|}{ Timpanoesclerosis } \\
\hline $\mathrm{Si}$ & 0 & 11 & $\mathrm{NC}$ & NC & $1.0^{\mathrm{a}}$ \\
\hline No & 5 & 70 & & & \\
\hline
\end{tabular}

RR: Riesgo relativo. NC: No calculable

a Prueba exacta de Fisher 
Tabla 5. Línea de base audiométrica (valores preoperatorios) en los sujetos del estudio.

\begin{tabular}{|l|r|r|r|}
\hline \multicolumn{1}{|c|}{$\begin{array}{c}\text { Variable } \\
\text { audiométrica } \\
(\mathbf{d B})\end{array}$} & $\begin{array}{c}\text { Cohorte } \\
\text { expuesta } \\
\mathbf{n = 4 3}\end{array}$ & $\begin{array}{c}\text { Cohorte no } \\
\text { expuesta } \\
\mathbf{n = 4 3}\end{array}$ & \multicolumn{1}{|c|}{$\mathbf{p}$} \\
\hline PTP vía aérea: Mediana [RIC] & $33.8[19.95]$ & $25.0[8.0]$ & $0.008^{\mathrm{a}}$ \\
\hline PTP vía ósea: Mediana [RIC] & $12.5[8.75]$ & $10.0[5.0]$ & $0.71^{\mathrm{a}}$ \\
\hline BAH: Mediana [RIC] & $20.0[11.3]$ & $15.0[10.0]$ & $<0.001^{\mathrm{a}}$ \\
\hline URH: Mediana [RIC] & $30.0[20.0]$ & $25.0[10.0]$ & $0.24^{\mathrm{a}}$ \\
\hline PDL: Mediana [RIC] & $100[0]$ & $100[0]$ & $1.0^{\mathrm{a}}$ \\
\hline
\end{tabular}

dB: decibeles. RIC: recorrido intercuartil. PTP: Promedio de tonos puros de 4 frecuencias. BAH = PTP vía aérea - PTP vía ósea. URH: umbral de recepción del habla. PDL: porcentaje de discriminación del lenguaje

a Prueba U de Mann-Whitney

Tabla 6. Resultado funcional dentro de cada cohorte

\begin{tabular}{|c|c|c|c|c|}
\hline \multirow{3}{*}{$\begin{array}{c}\text { Variable } \\
\text { audiométrica } \\
\text { (dB) }\end{array}$} & \multicolumn{2}{|c|}{ Cohorte expuesta } & \multicolumn{2}{|c|}{ Cohorte no expuesta } \\
\hline & $\begin{array}{c}\text { Preo } \\
\text { peratorio }\end{array}$ & $\begin{array}{c}\text { Post } \\
\text { operatorio }\end{array}$ & $\begin{array}{c}\text { Pre } \\
\text { operatorio }\end{array}$ & $\begin{array}{c}\text { Post } \\
\text { operatorio }\end{array}$ \\
\hline & \multicolumn{2}{|c|}{ p* } & \multicolumn{2}{|c|}{ p* } \\
\hline \multirow{2}{*}{$\begin{array}{l}\text { PTP vía aérea } \\
\text { Mediana [RIC] }\end{array}$} & 33.8 [19.95] & $21.3[11.7]$ & $25.0[8.0]$ & $15.0[15.0]$ \\
\hline & \multicolumn{2}{|c|}{$0.001^{\mathrm{a}}$} & \multicolumn{2}{|c|}{$<0.001^{\mathrm{a}}$} \\
\hline \multirow{2}{*}{$\begin{array}{l}\text { PTP vía ósea } \\
\text { Mediana [RIC] }\end{array}$} & $12.5[8.75]$ & $13.8[8.0]$ & $10.0[5.0]$ & $10[5.0]$ \\
\hline & \multicolumn{2}{|c|}{$0.38^{\mathrm{a}}$} & \multicolumn{2}{|c|}{$0.98^{\mathrm{a}}$} \\
\hline \multirow{2}{*}{$\begin{array}{l}\text { BAH } \\
\text { Mediana [RIC] }\end{array}$} & 20.0 [11.3] & $10.0[10.0]$ & $15.0[10.0]$ & $5.0[10.0]$ \\
\hline & \multicolumn{2}{|c|}{$<0.001^{\mathrm{a}}$} & \multicolumn{2}{|c|}{$<0.001^{\mathrm{a}}$} \\
\hline \multirow{2}{*}{$\begin{array}{l}\text { URH } \\
\text { Mediana [RIC] }\end{array}$} & $30.0[20.0]$ & $20.0[10]$ & $25.0[10.0]$ & $15.0[15.0]$ \\
\hline & \multicolumn{2}{|c|}{$0.006^{a}$} & \multicolumn{2}{|c|}{$0.001^{a}$} \\
\hline \multirow{2}{*}{$\begin{array}{l}\text { PDL } \\
\text { Mediana [RIC] }\end{array}$} & $100[0]$ & $100[0]$ & $100[0]$ & $100[0]$ \\
\hline & \multicolumn{2}{|c|}{$0.11^{\mathrm{a}}$} & \multicolumn{2}{|c|}{$1.0^{\mathrm{a}}$} \\
\hline
\end{tabular}

$\mathrm{dB}$ : decibeles. RIC: recorrido intercuartil. PTP: Promedio de tonos puros de 4 frecuencias. BAH: brecha aíre-hueso URH: umbral de recepción del habla. PDL: porcentaje de discriminación del lenguaje.

$\mathrm{BAH}=$ PTP vía aérea - PTP vía ósea

* Las comparaciones se hicieron entre el resultado preoperatorio y a los 6 meses postoperatorio, dentro de cada grupo.

a Prueba de los rangos con signo de Wilcoxon.

95\% $=1.19-2.59]$, utilizando como criterio de éxito una $\mathrm{BAH} 10 \mathrm{~dB}$. Si se utiliza como criterio de éxito una $\mathrm{BAH}$ $20 \mathrm{~dB}$ el resultado fue $\mathrm{RR}=1.28$ [IC 95\%=0.72-2.30].

No se presentaron complicaciones mayores ni en el postoperatorio inmediato ni tardío. Tres pacientes de la cohorte expuesta $(7 \%)$ y dos de la cohorte no expuesta $(4.7 \%)$
Tabla 7. Comparación del resultado funcional entre la cohorte expuesta y la no expuesta.

\begin{tabular}{|c|c|c|c|}
\hline $\begin{array}{c}\text { Variable } \\
\text { audiométrica } \\
\text { (dB) }\end{array}$ & $\begin{array}{c}\text { Cohorte } \\
\text { expuesta } \\
\text { Grupo I } \\
n=43\end{array}$ & \begin{tabular}{|c|} 
Cohorte no \\
expuesta \\
Grupo II \\
$n=43$
\end{tabular} & $\mathbf{p}$ \\
\hline \multicolumn{4}{|l|}{ Cierre BAH $10 \mathrm{~dB}$} \\
\hline No & 18 & 7 & \multirow[b]{2}{*}{$0.017^{\circ}$} \\
\hline $\mathrm{Si}$ & 25 & 36 & \\
\hline \multicolumn{4}{|l|}{ Cierre $\mathrm{BAH} 20 \mathrm{~dB}$} \\
\hline No & 5 & 3 & \multirow[b]{2}{*}{$0.71^{b}$} \\
\hline $\mathrm{Si}$ & 38 & 40 & \\
\hline $\begin{array}{l}\text { BAH postoperatorio } \\
\text { Mediana }[\mathrm{RIC}]\end{array}$ & $10[10]$ & $5[10]$ & $0.032^{\mathrm{c}}$ \\
\hline $\begin{array}{l}\text { Cierre de BAH } \\
\text { Mediana }[\mathrm{RIC}]\end{array}$ & $8[16.4]$ & $10[14]$ & $0.44^{c}$ \\
\hline
\end{tabular}

dB: decibeles. RIC: recorrido intercuartil. BAH: Brecha aíre-hueso $\mathrm{BAH}=\mathrm{PTP}$ vía aérea - PTP vía ósea. Cierre de BAH $=\mathrm{BAH}$ preoperatorio - $\mathrm{BAH}$ postoperatorio

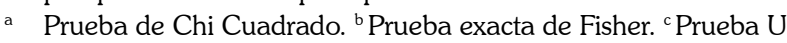
de Mann-Whitney.

presentaron otorrea en el primer mes del postoperatorio; todos ellos se manejaron con aspiración de oído y ciprofloxacina tópica, consiguiendo control de la infección. En su seguimiento estos cinco pacientes tuvieron neo tímpano integro en posición adecuada.

\section{Discusión}

Los resultados del estudio muestran que para la timpanoplastia tipo I los pacientes de la cohorte expuesta (trauma por onda explosiva) presentan resultados comparables a los de la cohorte no expuesta (otitis crónica) desde el punto de vista anatómico, aunque hubo peor resultado funcional en la cohorte expuesta $(\mathrm{RR}=1.76$. IC $\mathrm{RR} 95 \%=$ 1.19-2.59).

El estudio fue diseñado con el fin de comparar de forma prospectiva los resultados de la cohorte expuesta respecto a la cohorte no expuesta en el escenario clínico rutinario en que se manejan estos pacientes: un hospital de referencia de tercer nivel. Con los criterios de inclusión/exclusión utilizados se limitó la muestra a pacientes adultos con perforación timpánica aislada (sin patología mastoidea), utilizando la misma técnica quirúrgica y el mismo injerto en todos los casos. Estas características del diseño permitieron la eliminación de varias variables (factores de confusión) que pudieran afectar el análisis del resultado quirúrgico: colesteatoma (19), tipo de técnica quirúrgica $(2,20)$, tipo de injerto utilizado (18) y estado de la cadena oscicular (18-20). 
Una de las dificultades que se presentó fue la pérdida en el seguimiento en una parte de los pacientes (15/109, 13.8\%). Sin embargo, por el tipo de diseño utilizado el estudio se terminó una vez se completó el tamaño de muestra calculado (incrementado la duración prevista del estudio en un año), por lo cual la pérdida de los pacientes no afecta de forma importante la validez interna del estudio. Una variable que no se tuvo en cuenta en el presente estudio, y que podría explicar una parte de los fracasos quirúrgicos, fue el apego de los pacientes a las recomendaciones de cuidado postoperatorio. El resultado de la timpanoplastia se puede ver afectado por maniobras que modifiquen la presión en el oído medio respecto al oído externo, como pueden ser: estornudos con boca cerrada, cambios de altura sobre el nivel del mar, sonarse la nariz, ejercicio físico intenso; entre otros. Aunque a todos los pacientes del estudio se les entregó por escrito las mismas recomendaciones de cuidado postoperatorio (esto hace parte del protocolo de manejo de esta cirugía en el servicio de Otorrinolaringología de la institución del estudio), no se monitorizó el cumplimiento de las mismas por parte de los pacientes.

Aunque hubo diferencias entre la cohorte expuesta y la no expuesta respecto a: sexo, tamaño de la perforación timpánica y localización anterior de la perforación timpánica (Tablas 1 y 3); el análisis bivariado mostró que estas variables clínicas no afectaron el resultado quirúrgico (Tabla 4). Tampoco hubo asociación entre la integración del injerto y la inflamación/infección al momento de cirugía y la timpanoesclerosis (Tabla 4).Estos hallazgos apuntan a que los grupos son comparables con respecto a la línea base.

Los porcentajes de éxito anatómico encontrados en el presente estudio (cohorte expuesta $=90.7 \%$; cohorte no expuesta $=97.7 \%$ ), son similares a los reportados en otros estudios en otitis crónica $(2,16,18)$ y en víctimas de trauma por onda explosiva (13). Al igual son congruentes con lo reportado en un estudio previo de auditoría clínica de resultados hecho en la misma institución del presente estudio (19). Estos resultados no apoyan la hipótesis de que los factores que afectan negativamente el cierre espontáneo en una perforación timpánica traumática causada por trauma por onda explosiva (con respecto al encontrado para otras etiologías), influyan en el éxito anatómico de la cirugía. Con respecto a la posibilidad de lesión térmica en el remanente timpánico, con la posible disminución en la capacidad reparativa, consideramos que el reavivamiento de los bordes de la perforación (Figura 1b) es una maniobra suficiente para exponer tejido epitelial con la suficiente vitalidad para crecer sobre el injerto utilizado en la cirugía $(1,2)$. Respecto a la sobreinfección y perforaciones mayo- res, los resultados de este estudio muestran que estas no variables no se asocian con el resultado anatómico. Resultados similares para estas dos variables han sido descritos en estudios previos (20). Consideramos que siempre que se ejecute una técnica quirúrgica adecuada, se pueden obtener buenos resultados independientemente del tamaño de la perforación de la MT y de la presencia de inflamación/infección al momento de la cirugía.

Con respecto al resultado funcional se encontró un porcentaje de éxito menor en la cohorte expuesta (58.1\%), respecto a la cohorte no expuesta (83.7\%); cuando se utilizó como criterio de éxito una $\mathrm{BAH} 10 \mathrm{~dB}$. El riesgo relativo de fracaso funcional en la cohorte expuesta fue de 1.76 [IC 95\% RR=1.19-2.59]. Dado que los estudios previos que los autores encontramos al revisar la literatura en pacientes victimas de trauma por onda explosiva (PubMed, términos: "Tympanoplasty" [Mesh] AND "Tympanic Membrane Perforation "[Mesh]; sin límites. Búsqueda hecha en febrero 25 de 2011), no utilizan el resultado funcional como criterio de éxito (13); se limitan las comparaciones que se podrían hacer en esta población. Sin embargo, en un estudio en que no se discrimina los resultados por la etiología de la perforación timpánica (incluyó pacientes con otitis crónica y trauma por onda explosiva), se reportan un cierre de la $\mathrm{BAH} \quad 10 \mathrm{~dB}=74.5 \%$ (19); que estaría en un punto medio entre las dos cohortes reportadas en el presente estudio. Otro estudio reporta un cierre de la $\mathrm{BAH} 20 \mathrm{~dB}=76.7 \%$ (21), en timpanoplastia revisional, que es inferior al que reportamos para la cohorte expuesta (88.4\%) y la cohorte no expuesta (93\%). Aunque el criterio de $\mathrm{BAH} 10 \mathrm{~dB}$ es el recomendado como punto de corte para evaluar el éxito funcional (17), un punto de corte en $20 \mathrm{~dB}$ tiene un importante sentido práctico. Cuando en el postoperatorio un paciente tiene un neo tímpano íntegro (éxito anatómico) pero presenta una $\mathrm{BAH}$, se considera que es candidato para cirugía funcional adicional si la $\mathrm{BAH}$ $>20 \mathrm{~dB}$; de lo contrario (BAH 0-20 dB) no se recomienda cirugía funcional. Utilizando el punto de corte de $20 \mathrm{~dB}$ para el éxito funcional, no hubo diferencias significativas entre las cohortes expuesta y no expuesta; y los porcentajes de éxito son comparables a los reportados en estudios previos que no discriminan por la etiología de la perforación timpánica $(19,21)$.

Respecto las razones para el peor resultado funcional observado en la cohorte expuesta, creemos que deben estar en relación con problemas de motilidad de la cadena oscicular. Dichos problemas de motilidad (fijación o disrupción) deben ser parciales, ya que como grupo observamos un cierre de la $\mathrm{BAH} \quad 20 \mathrm{~dB}$ del $88.4 \%$ para la cohorte 
expuesta y del 93\% para la cohorte no expuesta. Como se mencionó, una disrupción completa o fijación completa de la cadena oscicular genera $\mathrm{BAH}>20 \mathrm{~dB}$ (22); y en tales pacientes se recomienda cirugía funcional adicional. Pero, ¿Cuáles serían los posibles mecanismos de fijación/ disrupción parcial de la cadena oscicular en pacientes con perforación timpánica secundaría a trauma por onda explosiva? La cantidad de energía liberada por los artefactos explosivos pueden causar además de la perforación timpánica, daños a la mucosa del oído medio cuyo proceso reparativo generaría bridas/fibrosis mucosa que restringe la motilidad oscicular. En la cohorte expuesta se encontró que la distancia promedio al punto de la explosión fue $3.1 \mathrm{~m}$ y que $34.9 \%$ de los pacientes tuvieron otras lesiones corporales; lo que sugiere que estos pacientes estuvieron expuestos a importantes cantidades de energía (7). El hecho de que el tamaño de la perforación timpánica fuera significativamente mayor en la cohorte expuesta, también apoya la anterior afirmación.

El porcentaje de trauma acústico en las frecuencias del habla en la cohorte expuesta fue del $44.2 \%$. Este porcentaje es inferior a lo reportado por otros autores del $80 \%$ (11) y $70.6 \%$ (14). Sin embargo debemos aclarar que usamos como definición de trauma acústico una alteración en el PTP de cuatro frecuencias $(0.5-3 \mathrm{KHz})$, a diferencia de los otros autores que reportan la alteración en frecuencias agudas $(4-8 \mathrm{KHz})$, por lo cual los anteriores porcentajes no son directamente comparables. De todas formas se observa que hay un daño neurosensorial en un importante porcentaje de pacientes.

En conclusión, los resultados del presente estudio muestran que el resultado anatómico de la timpanoplastia tipo I es equiparable entre los pacientes con perforación timpánica secundaria a trauma por onda explosiva respecto a aquellos con perforaciones timpánicas por otitis crónica. Sin embargo se observó un peor resultado funcional entre los pacientes víctimas de trauma por onda explosiva, cuyos mecanismos están aún por aclararse, pero que tendrían relación con una mayor predisposición a fijación/ disrupción parcial de la cadena oscicular. También está pendiente por establecer el papel del apego a los cuidados postoperatorios, que no fue estudiado en el presente estudio.

Agradecimientos: Al Hospital Militar Central, por el apoyo institucional para la realización de la presente investigación.

Conflicto de intereses: Los autores declaran no tener ningún conflicto de intereses.
Financiación: Proyecto con financiación del Hospital Militar Central, Proyecto C-02-2011, según la primera convocatoria-concurso: "Conformación de un banco de anteproyectos de investigación académica en el Hospital Militar Central Año 2011 y selección de protocolos para financiación". Resolución No. 1178 del 19 de octubre de 2011.

\section{Referencias}

1. Rizer FM. Overlay versus underlay tympanoplasty. Part I: historical review of the literature. Laryngoscope. 1997;107(12 Pt 2):1-25.

2. Rizer FM. Overlay versus underlay tympanoplasty. Part II: the study. Laryngoscope. 1997;107(12 Pt 2):26-36.

3. Mrena R, Pââkkônen L, Bâck L, et al. Otologic Consequences of Blast Exposure: A Finnish Case Study of Shopping Mall Bomb Explosion. ActaOtolaryngol 2004;124:946-952.

4. Felipe EE, Cabrera JG, Ordoñez LE, et al. Perforación de la membrana timpánica asociada a trauma por explosivos. Acta de Otorrinolaringología y Cirugía de Cabeza y Cuello. 2010;38:295-300.

5. Harrison CD, Bebarta VS, Grant GA. Tympanic membrane perforation after combat blast exposure in Iraq: a poor biomarker of primary blast injury. J Trauma 2009;67:210-211.

6. Persaud R, Hajioff D, Wareing M, et al. Otological trauma resulting from the Soho Nail Bomb in London; April 1999. Clin Otolaryngol. 2003;28:203-206.

7. Champion HR, Holcomb JB, Young LA. Injuries from explosions: Physics, Biophysics, Pathology, and Required Research Focus. J Trauma. 2008;66:1468-1477.

8. DePalma RG, Burris DG, Champion HR, et al. Blast Injuries. N Engl J Med. 2005;352:1335-1342.

9. Orji FT, Agu CC. Determinants of spontaneous healing in traumatic perforations of the tympanic membrane. ClinOtolaryngol. 2008;33:420-426.

10. Kristensen S. Spontaneous healing of traumatic tympanic membrane perforations in man: a century of experience. J Laryngol Otol. 1992;106:1037-50.

11. Ritenour AE, Wickley A, Ritenour JS, et al. Tympanic membrane perforation and hearing loss from blast overpressure in Operation Enduring Freedom and Operation Iraqi Freedom wounded. J Trauma. 2008;64 (2 Suppl):S174-8.

12. Miller IS, McGahey D, Law K. The Otologic consequences of the Omagh bomb disaster. Otolaryngol Head Neck Surg. 2002;126:127-8.

13. Sprem N, Branica S, Dawidowsky K. Tympanoplasty after war blast lesions of the eardrum: retrospective study. Croat Med J. 2001;42:642-5.

14. Cohen JT, Ziv G, Bloom J, et al. Blast injury of the ear in a confined space explosion: auditory and vestibular evaluation. Isr Med Assoc J. 2002;4:559-62.

15. von Elm E, Altman DG, Egger M, et al. The Strengthening the Reporting of Observational Studies in Epidemiology (STROBE) 
statement: guidelines for reporting observational studies. Lancet. 2007;370:1453-7.

16. Kartush JM, Michaelides EM, Becvarovski Z, et al. Over-under tympanoplasty. Laryngoscope. 2002;112:802-7.

17. Committee on Hearing and Equilibrium guidelines for the evaluation of results of treatment of conductive hearing loss. American Academy of Otolaryngology-Head and Neck Surgery Foundation, Inc. Otolaryngol Head Neck Surg. 1995;113: 186-7.

18. Abstracts of the annual meeting of the American Academy of Otolaryngology-Head and Neck Surgery Foundation, September 26-29, 2010. Boston, Massachusetts, USA. Graft choice in tympanoplasty: temporalis fascia vs. auricular cartilage. Otolaryngol Head Neck Surg 2010;143(2 Suppl 2):P94-95.
19. Ordóñez-Ordóñez LE, Prieto-Rivera JA, Guzmán-Durán JE, et al. Tympanomastoid surgery for chronic ear: clinical audit. Acta de Otorrinolaringología \& Cirugía de Cabeza y Cuello. 2008; 36:19199.

20. Ordóñez-Ordóñez LE, Angulo-Martínez ES, Prieto-Rivera JA, et al. Risk factors leading to failure in myringoplasty: a case-control study. Acta Otorrinolaringol Esp. 2008; 59: 176-82.

21. Altuna X, Navarro JJ, Algaba J. Island cartilage tympanoplasty in revision cases: anatomic and functional results. Eur Arch Otorhinolaryngol. 2012; 269:2169-72.

22. Merchant SN, Ravicz ME, Puria $\mathrm{S}$ et al. Analysis of middle ear mechanics and application to diseased and reconstructed ears.Am J Otol. 1997;18:139-54. 